\title{
Gut microbiota dysbiosis contributes to the development of chronic obstructive pulmonary disease
}

Naijian $\mathrm{Li}^{1 \dagger}$, Zhouli Dai ${ }^{2+}$, Zhang Wang ${ }^{3}$, Zhishan Deng ${ }^{1}$, Jiahuan Zhang ${ }^{1}$, Jinding Pu' ${ }^{1}$, Weitao Cao ${ }^{1}$, Tianhui Pan' ${ }^{1}$ Yumin Zhou' ${ }^{1}$ Zhaowei Yang ${ }^{1}$, Jing Li ${ }^{1}$, Bing Li ${ }^{4}$ and Pixin Ran ${ }^{1 *}$ (D)

\begin{abstract}
Background: Dysbiosis of the gut microbiome is involved in the pathogenesis of various diseases, but the contribution of gut microbes to the progression of chronic obstructive pulmonary disease (COPD) is still poorly understood.

Methods: We carried out 165 rRNA gene sequencing and short-chain fatty acid analyses in stool samples from a cohort of 73 healthy controls, 67 patients with COPD of GOLD stages I and II severity, and 32 patients with COPD of GOLD stages III and IV severity. Fecal microbiota from the three groups were then inoculated into recipient mice for a total of 14 times in 28 days to induce pulmonary changes. Furthermore, fecal microbiota from the three groups were inoculated into mice exposed to smoke from biomass fuel to induce COPD-like changes.

Results: We observed that the gut microbiome of COPD patients varied from that of healthy controls and was characterized by a distinct overall microbial diversity and composition, a Prevotella-dominated gut enterotype and lower levels of short-chain fatty acids. After 28 days of fecal transplantation from COPD patients, recipient mice exhibited elevated lung inflammation. Moreover, when mice were under both fecal transplantation and biomass fuel smoke exposure for a total of 20 weeks, accelerated declines in lung function, severe emphysematous changes, airway remodeling and mucus hypersecretion were observed.
\end{abstract}

Conclusion: These data demonstrate that altered gut microbiota in COPD patients is associated with disease progression in mice model.

Keywords: COPD, Gut microbiome, Fecal transplant, Mice, Lung inflammation

\section{Background}

Chronic obstructive pulmonary disease (COPD) is a worldwide public health concern and a leading cause of morbidity and mortality $[1,2]$. Currently, bronchodilator and anti-inflammatory therapies are still the mainstay

\footnotetext{
*Correspondence: pxran@gzhmu.edu.cn

${ }^{\dagger}$ Naijian Li and Zhouli Dai contributed equally to the work

${ }^{1}$ Department of Allergy and Clinical Immunology, State Key Laboratory

of Respiratory Disease, National Clinical Research Center for Respiratory

Disease, Guangzhou Institute of Respiratory Health, The First Affiliated

Hospital of Guangzhou Medical University, 151 Yanjiang Road,

Guangzhou, Guangdong, People's Republic of China

Full list of author information is available at the end of the article
}

of pharmacological treatment in COPD [3]. Although progress has been made in the treatment of symptoms and prevention of acute exacerbations, few advances have been achieved in ameliorating disease progression or affecting mortality $[2,4]$. COPD is a complex disease with multiple sub-phenotypes that affects not only the lungs but also the cardiovascular, gastrointestinal, and immune systems, and the underlying mechanisms are not completely understood [5-7].

The role of the microbiome, and the gut microbiome in particular, has drawn considerable attention in human health and disease. Emerging evidence has revealed the original author(s) and the source, provide a link to the Creative Commons licence, and indicate if changes were made. The images or other third party material in this article are included in the article's Creative Commons licence, unless indicated otherwise in a credit line to the material. If material is not included in the article's Creative Commons licence and your intended use is not permitted by statutory regulation or exceeds the permitted use, you will need to obtain permission directly from the copyright holder. To view a copy of this licence, visit http://creativecommons.org/licenses/by/4.0/. The Creative Commons Public Domain Dedication waiver (http://creativeco mmons.org/publicdomain/zero/1.0/) applies to the data made available in this article, unless otherwise stated in a credit line to the data. 
role of gut microbiota dysbiosis in various chronic human diseases $[8,9]$. Moreover, by fecal microbiota transplantation (FMT) experiments in animals and in humans, the causal relationships between the gut microbiota and the pathogenesis of multiple diseases in humans have been demonstrated. For example, gut microbiota dysbiosis contributes to the development of hypertension, and the short-chain fatty acid (SCFA) propionate, which is produced from dietary fiber by gut bacteria, has been shown to ameliorate hypertensive end-organ damage $[10,11]$. Alteration of the gut microbiota by the antibiotic agent azithromycin reduces airway inflammation in allergic asthmatic patients [12]. However, little is known about the involvement of the gut microbiota in COPD. Although primarily considered a respiratory disease, COPD commonly co-occurs with chronic gastrointestinal tract diseases [7, 13]. Recent studies have linked changes in the gut microbial composition and function to disease development in the lungs, but knowledge of the gut-lung axis in COPD is scarce $[14,15]$. The features of the gut microbiota in patients with COPD remain to be determined and the contribution of gut microbes to disease progression is still poorly understood.

In the present study, we performed $16 \mathrm{~S}$ ribosomal RNA sequencing of stool samples from COPD patients and healthy controls, described the features of the gut microbiota, and analyzed their SCFA profiles. Next, we performed fecal microbiota transplantation into mice, demonstrating the role of disordered gut microbiome in COPD.

\section{Methods \\ Study design}

To investigate the features of the gut microbiota in COPD patients and the contribution of the gut-lung axis in COPD, we designed a systematic and reproducible workflow (Fig. 1a-c). All subjects in the present study were recruited from the 135 Key Research and Development Program (No._2016YFC1304101), which is a populationbased, cross-sectional, multicenter survey of COPD conducted in China. In our previous COPD studies in this cohort [16-18], we obtained a representative sample of

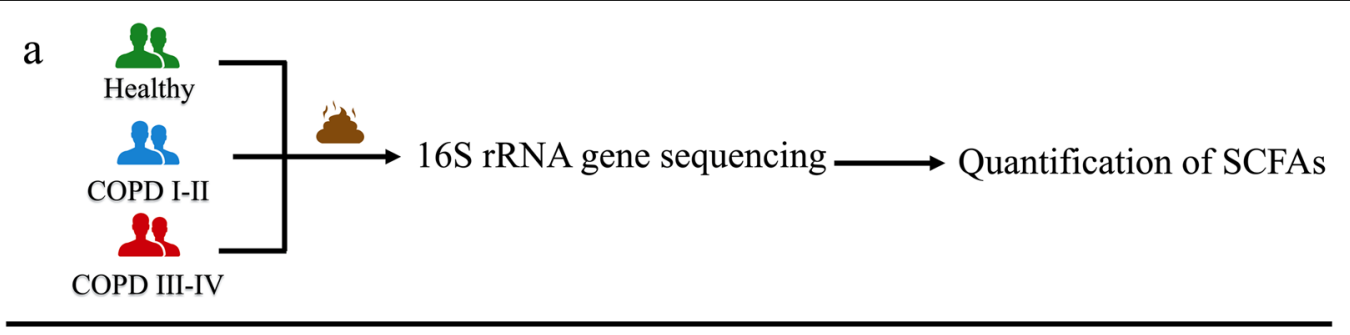

b
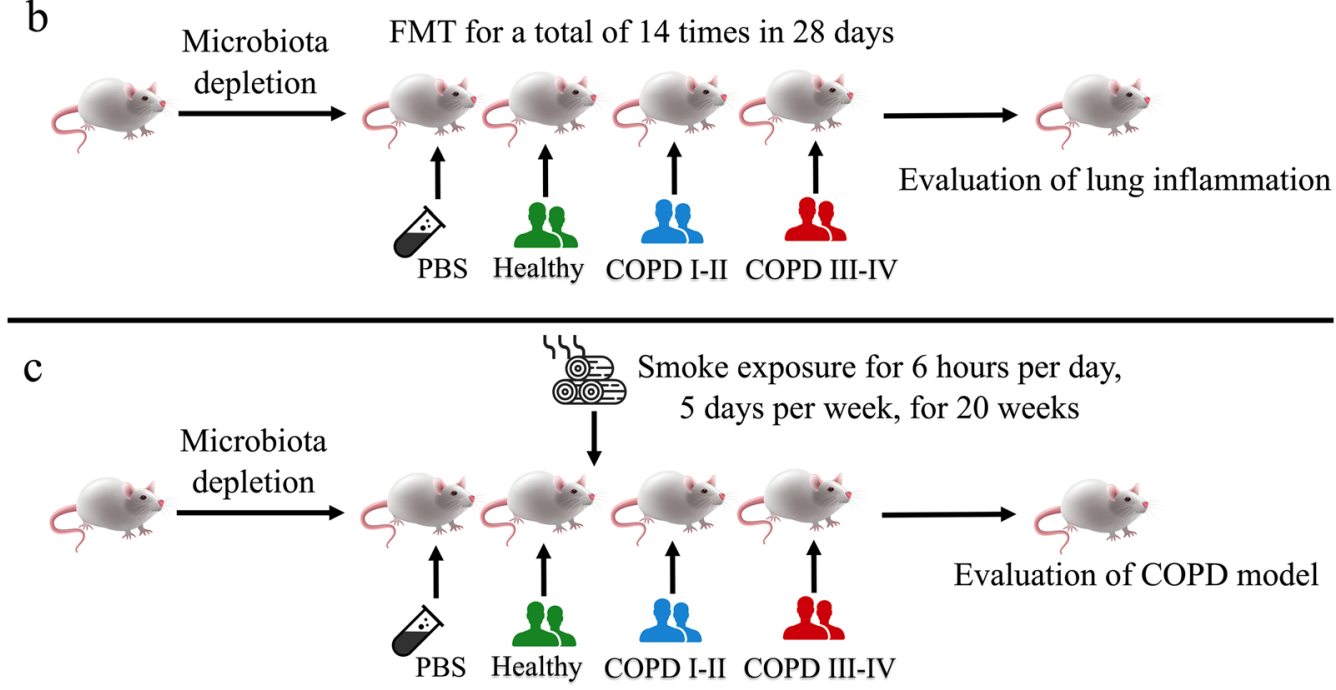

FMT for a total of 40 times in 20 weeks

Fig. 1 Schematic overview of the study workflow. a Healthy individuals $(n=73)$ and COPD patients $(n=99)$ were recruited for the study. Stool samples were subjected to $16 \mathrm{~S}$ ribosomal RNA sequencing and short-chain fatty acid (SCFA) profiling. $\mathbf{b}$ Fecal microbiota from healthy individuals and from COPD patients were pooled and inoculated into mice by fecal microbiome transplantation for inflammatory phenotyping. $\mathbf{c}$ During the biomass fuel exposure, the fecal microbiota transplantation was performed 
the COPD population in Wengyuan and Guangzhou, and the healthy group comes from the same village or residential area. All patients were residents of Wengyuan and Guangzhou, which are approximately $130 \mathrm{~km}$ apart and characterized by similar lifestyles and eating habits. All recruited patients and healthy subjects provided written informed consent before stool donation.

\section{Study cohort and patient characteristics}

All participants underwent pulmonary function measurements to diagnose COPD according to GOLD guidelines [3]. Spirometry data were obtained as previously described [18]. The participants were classified by pulmonary function as described in GOLD guidelines. The final cohort was composed of 73 healthy controls $\left(\mathrm{FEV}_{1} / \mathrm{FVC}\right.$ ratio $\geq 70 \%$ and $\mathrm{FEV}_{1} \geq 80 \%$ ), 67 patients with $\mathrm{COPD}$ GOLD severity stages I and II $\left(\mathrm{FEV}_{1} / \mathrm{FVC}\right.$ ratio $<70 \%$ and $\mathrm{FEV}_{1} 50-80 \%$ ), and 32 patients with COPD severity stages III and IV $\left(\mathrm{FEV}_{1} / \mathrm{FVC}\right.$ ratio $<70 \%$ and $\left.\mathrm{FEV}_{1}<50 \%\right)$.

All individuals underwent medical tests prior to stool sample collection, including chest X-ray, electrocardiography, abdominal ultrasound, and blood, urine, and stool tests. Subjects were required to meet the following criteria to qualify for the study: (1) Able and willing to provide written informed consent and medical records from the preceding year. (2) Male aged 40-80 years. (3) Diagnosed with COPD more than 1 year prior to the study. Subjects who met any of the following criteria prior to enrollment were excluded from the study: (1) Treatment with systemic (e.g., oral, intravenous, or intramuscular) corticosteroids within the preceding 8 weeks. (2) History of cystic fibrosis, asthma, and/or other clinically significant lung disease other than COPD. (3) A diagnosis of cancer, heart failure, hypertension, diabetes, infectious diseases, renal or liver dysfunction, gastrointestinal disease, or treatment with antibiotics (inclusive of macrolide antibiotics) within the preceding 8 weeks. All clinical information was collected according to standard procedures by the State Key Laboratory of Respiratory Disease of Guangzhou Medical University (Guangzhou, China).

\section{Stool sample collection and sequencing}

Fresh stool from donors was collected in the morning. The consistency of each sample was graded according to the Bristol Stool Form Scale, and only sample types 2-5 were included [19]. Stool samples in sterile containers were snap-frozen in dry ice and stored after arrival at the research laboratory in $-80{ }^{\circ} \mathrm{C}$ freezers until processing. Total bacterial DNA extraction of stool was carried out on $100 \mathrm{mg}$ of sample using the HiPure Stool DNA kits (Magen BioSciences, Waltham, MA, USA) in accordance with the manufacturer's instructions. The extracted DNA from each sample was used as a template to amplify the V3-V4 region of $16 \mathrm{~S}$ rRNA genes using PCR. 16S rRNA gene amplification, in vitro transcription and labeling, and hybridization were conducted using the Illumina 16S Metagenomic Sequencing Library preparation guide [20]. 16S amplicon PCR forward primer 5'-(TCG TCG GCA GCG TCA GAT GTG TAT AAG AGA CAG CCT ACG GGN GGC WGC AG) $-3^{\prime}$ and $16 \mathrm{~S}$ amplicon PCR reverse primer $5^{\prime}$-(GTC TCG TGG GCT CGG AGA TGT GTA TAA GAG ACA GGA CTA CHV GGG TAT CTA ATC C) $-3^{\prime}$ were used [21]. All libraries were sequenced by using an Illumina MiSeq PE250 platform (San Diego, CA, USA) at the RiboBio Genome Center (Guangzhou, China).

\section{Gut microbiota analysis}

16S rRNA gene sequence analysis, including raw sequence filtering and taxonomic classification, was performed as described previously [22]. Briefly, the raw sequencing data were filtered for quality (Q30) and joined by FLASH ( http://ccb.jhu.edu/software/FLASH/) [23]. Sequences that contained read lengths shorter than $200 \mathrm{bp}$ were removed. The QIIME (Quantitative Insights into Microbial Ecology, v1.9.1, http://qiime.org/) software pipeline was used to cluster high-quality reads into operational taxonomic units (OTUs) at the $97 \%$ identity level. OTU search was performed using the GreenGenes 13.8 database. The $\alpha$-diversity indices (Chao1 index) was calculated by QIIME. The bar diagram of alpha diversity indices and relative abundance were drawn using GraphPad Prism 8 software (GraphPad Software Inc., San Diego, CA, USA).

Enterotyping was run with a reference-based online tool (http://enterotypes.org) as indicated by Costea et al. [24]. Only genera with an average relative abundance $\geq 10^{-4}$ and that appeared in at least $50 \%$ of samples in each group were considered in the analysis. Pearson's Chi-squared test was used for testing enterotypes between clinical groups. Significance of community dissimilarity based on Bray-Curtis dissimilarity matrices was tested using a permutational multivariate analysis of variance (PERMANOVA) function (adonis) within the $R$ package vegan.

\section{Quantification of SCFAs in stool samples}

Seven SCFAs (acetic, propionic, butyric, isobutyric, valeric, isovaleric, and caproic acids) were measured in the stool samples by high-performance gas chromatography (Agilent 6890N; Agilent Technologies, Santa Clara, CA, USA) with an autosampler and a flame ionization detector according to the manufacturer's guidelines. 


\section{Animal experiments \\ Mice}

Specific pathogen-free male C57BL/6 mice were purchased from Guangzhou University of Chinese Medicine (Guangzhou, China). The mice were housed five to a cage. All experiments were conducted with mice with 8-10 weeks of age. Temperature and relative humidity in the animal facility were controlled at $23 \pm 2{ }^{\circ} \mathrm{C}$ and at $40-70 \%$, respectively. Lighting was artificial with a sequence of $12 \mathrm{~h}$ of light (06:00-18:00) and $12 \mathrm{~h}$ of darkness. Rodent food pellets and water were sterilized and provided ad libitum. The Animal Medical Center of Guangzhou Medical University reviewed and approved all experiments (identification number: GY2018-084).

\section{Microbiota depletion and fecal transplantation}

To deplete the gut microbiota, mice were provided with broad-spectrum antibiotics (ampicillin $1 \mathrm{~g} / \mathrm{L}$; neomycin sulfate $1 \mathrm{~g} / \mathrm{L}$; metronidazole $1 \mathrm{~g} / \mathrm{L}$; vancomycin $0.5 \mathrm{~g} / \mathrm{L}$, all purchased from Sigma-Aldrich, St. Louis, MO, USA) in drinking water for 3 weeks as previously described [25]. Treatment with broad-spectrum antibiotics in drinking water was stopped 2 days before fecal microbiota transplantation. $1 \mathrm{~g}$ fecal matter from each human group was mixed and then resuspended in $4 \mathrm{~mL}$ of phosphate-buffered saline and homogenized. The homogenate was centrifuged at $200 \times g$ for $10 \mathrm{~min}$ at $4{ }^{\circ} \mathrm{C}$, the supernatant was collected and stored at $-80{ }^{\circ} \mathrm{C}$ for subsequent use. Enumeration of inoculum from healthy group, COPD I-II group and COPD III-IV group in $1 \mathrm{~mL}$ of initial suspension were performed according to plate count methods [26].

\section{Gut microbiota-induced murine model of pulmonary inflammation}

A total of 60 mice were randomly divided into four groups of 15: Phosphate-buffered saline (PBS) FMT group, healthy FMT group, COPD I-II patients FMT group, and COPD III-IV patients FMT group. After microbiota depletion, the fecal microbiota transplantation was performed by a single oral administration of 100 $\mu \mathrm{L}$ per mouse every other day, for a total of 14 times in 28 days. The mice received fecal transplants from healthy individuals, COPD I-II subjects, or COPD III-IV subjects, and PBS as control.

\section{Fecal transplantation experiment in biomass smoke-induced murine model of COPD}

Similar to the previous, a total of 60 mice were also randomly divided into four groups of 15: PBS FMT group, healthy FMT group, COPD I-II patients FMT group, and COPD III-IV patients FMT group. After microbiota depletion, all mice were exposed to smoke produced by smoldering China fir sawdust (40 g/exposure) for two 3-h periods, 5 days per week, for 20 weeks in an inhalation chamber (model INH-WB_NOE (R/M)_CAP (PM2.5)_CS_SP; TSE Systems, Bad Homburg, Germany) [27]. Particulate matter mass concentrations, particle size distributions, and gas concentrations (oxygen, carbon monoxide, nitrogen oxides, and sulfur dioxide) were monitored by a DustTrak II aerosol monitor 8530 (TSI, Shoreview, MN, USA) and a Testo 340 portable flue gas analyzer (Testo, Lenzkirch, Germany) in the exposure rooms. During the biomass smoke exposure, the fecal microbiota transplantation was performed by a single oral administration of $100 \mu \mathrm{L}$ per mouse twice a week, for a total of 40 times in 20 weeks.

\section{Measurement of lung function}

Spirometry data were obtained as previously described using a Forced Pulmonary Maneuver System (Buxco Research Systems, Wilmington, NC, USA) [28]. Mice were sedated with $3 \%$ pentobarbital $(1 \mathrm{~mL} / \mathrm{Kg})$, and were tracheostomized and intubated, then the mice placed supine in the body chamber and connected to the system. The depth of anesthesia was maintained at a light surgical plane for the duration of testing, and the dose could be adjusted as necessary. The Cchord (chord compliance, between 0 and $10 \mathrm{~cm} \mathrm{H}_{2} 0$ ), $\mathrm{FEV}_{20}$ (forced expiratory volume in $20 \mathrm{~s}$ ), FRC (functional residual capacity), PEF (peak expiratory flow), MMEF (mean mid expiratory flow) and minute ventilation volume were measure within $10 \mathrm{~min}$. At least three acceptable maneuvers for each test of every mice were conducted to obtain a reliable mean spirometry data.

\section{Bronchoalveolar lavage fluid differential cell count and biomarker analysis}

Mice were sacrificed by $\mathrm{CO}_{2}$ and lung tissue and blood samples were collected. Whole lungs were cyclically inflated and deflated with $1 \mathrm{~mL}$ of phosphate-buffered saline (Gibco-Thermo Fisher Scientific, Waltham, MA, USA) three times. Cells were isolated by centrifugation at $300 \times g$ for $10 \mathrm{~min}$ at $4{ }^{\circ} \mathrm{C}$ and stained with Diff-Quik stain (Baso Diagnostics, Zhuhai, China). Differential cell counts were assessed from 400 cells counted on each slide. Plasma cytokine levels were assayed using Luminex xMap and a commercially available mouse cytokine 6-plex panel (Bio-Rad, Hercules, CA, USA) according to the manufacturer's guidelines and measured on a BioPlex 200 Platform.

\section{Flow cytometry}

Flow cytometry immunophenotyping was performed on T cells and B cells in whole blood. Peripheral blood 
mononuclear cells were isolated from sodium heparintreated venous blood samples by Ficoll-Hypaque density gradient and centrifugation at $1000 \times g$ at room temperature for $20 \mathrm{~min}$. We blocked the Fc receptors by incubating cells first with anti-CD16/32 antibodies for $15 \mathrm{~min}$ on ice. Cells were stained with the following antibodies for $30 \mathrm{~min}$ at room temperature: BB600-conjugated antimouse CD3, APC-conjugated anti-mouse CD19, BV421conjugated anti-mouse CD8, and FITC-conjugated anti-mouse CD4 (BD Biosciences, Franklin lakes, NJ, USA). After washing, cells were fixed with fluorescenceactivated cell sorting lysing solution (BD Biosciences). Appropriate isotype controls were used to determine the specificity of the staining. Flow cytometry data were acquired using FACSVerse (BD Biosciences) and analyzed with the FlowJo software (Tree Star, Inc., Ashland, OR, USA).

\section{Protein isolation and western blot assay}

Lung tissues were homogenized on ice for analysis of MUC2, MUC5AC, the tight junction protein claudin 1, $\alpha$ smooth-muscle actin (a-SMA), matrix metalloproteinase 2 (MMP-2), and neutrophil elastase levels. Total proteins were extracted from $100 \mathrm{mg}$ of lung tissues from each group, and concentrations were determined by the $\mathrm{BCA}$ protein assay. Thirty micrograms of total protein were loaded into each well and fractionated on a $10 \%$ SDS polyacrylamide gel. The housekeeping gene $\beta$-tubulin was used as an internal control to assess equal loading of total protein between wells. The bound antibodies were visualized using SuperSignal West Femto Maximum Sensitivity Substrate (Thermo Fisher Scientific). The abundance of target proteins was quantified by enhanced chemiluminescence.

\section{Pathology and immunohistochemistry}

Lung tissues were fixed with $4 \%$ paraformaldehyde solution and embedded in paraffin using standard methods as described previously [29]. Consecutive sections (3-5 $\mu \mathrm{m})$ were prepared, mounted on glass slides, and stained with hematoxylin and eosin. All slides were scanned and analyzed using an image analyzer platform (Leica, Wetzlar, Germany). Lung sections were stained with Alcian blue-periodic acid-Schiff (AB-PAS) using commercial kits (Sigma-Aldrich, St. Louis, MO, USA). The sections were incubated with primary antibodies against a-SMA, MMP-2, or MUC5AC (Abcam; Cambridge, UK). Quantitative analysis of IHC staining was performed using Image-Pro Plus 6.0 (Media Cybernetics, Bethesda, MD, USA). The alveolar destruction and the bronchial wall thickness were quantified as previously described [30]. Bronchial wall thickness was calculated as wall thickness $=($ total bronchial area - lumen area $) /$ total bronchial area, and the alveolar enlargement and destruction were quantified by the mean linear intercept (Lm). Sectioning and staining were performed by the Pathology Center of the First Affiliated Hospital of Guangzhou Medical University.

\section{Statistical analysis}

In the human cohort, microbiota data and SCFA levels were tested by ANOVA and the Kruskal-Wallis $\mathrm{H}$ test. The $p$ values were corrected for multiple testing using the Bonferroni method. Analyses were adjusted by age and smoking history. Pearson's $\chi^{2}$ test was used for testing enterotypes between clinical groups. Significance of community dissimilarity from Bray-Curtis dissimilarity matrices was tested using a permutational multivariate analysis of variance function (adonis) within the $\mathrm{R}$ package vegan. For animal experiments, comparisons were performed using ANOVA and $p$ values were also corrected using the Bonferroni method. Statistical analysis was performed in SPSS version 24 (IBM SPSS, Armonk, NY, USA), and the corrected $p<0.05$ was considered significant.

\section{Results}

\section{Characteristics of the study population}

A total of 172 Chinese participants were enrolled and divided into healthy controls $(n=73)$, COPD stage I-II $(\mathrm{n}=67)$, and COPD stage III-IV $(\mathrm{n}=32)$. Table 1 summarizes the characteristics of study participants. The subjects in the COPD group had lower body mass index, forced expiratory volume in $1 \mathrm{~s}$, forced vital capacity, and the forced expiratory volume in $1 \mathrm{~s}$ to forced vital capacity ratio but higher smoking index and COPD assessment scores than subjects in the control group. No significant difference was observed in occupation among the three groups.

\section{Altered gut microbial diversity and composition in COPD patients}

To assess whether gut microbial changes are associated with COPD severity, we performed 16S rRNA gene sequencing of fecal samples. Comparison between the three groups showed that intra-individual diversity, as measured by the number of operational taxonomic units (OTU), was significantly lower in the COPD III-IV group $(p=0.049$ vs. healthy subjects and $p=0.009$ vs. COPD I-II group, Fig. 2a). At the phylum level, all gut microbiota samples from the healthy group, COPD I-II group, and COPD III-IV group contained four major bacterial phyla: Bacteroidetes, Firmicutes, Proteobacteria, and Actinobacteria. The first three phyla accounted for over $96 \%$ of the total sequences in all three groups. The COPD III-IV group had a lower relative abundance of 
Table 1 Demographic characteristics of the study cohorts

\begin{tabular}{|c|c|c|c|c|}
\hline Characteristics & Healthy $(n=73)$ & COPD I-II $(n=67)$ & COPD III-IV $(n=32)$ & $p$ value \\
\hline Age, years & $59.7 \pm 9.4$ & $62.7 \pm 6.7$ & $61.5 \pm 6.6$ & NS \\
\hline $\mathrm{BMI}, \mathrm{kg} / \mathrm{m}^{2}$ & $24.6 \pm 2.9$ & $22.7 \pm 3.6$ & $20.8 \pm 2.7$ & $<0.01^{\mathrm{abc}}$ \\
\hline Smoking index (pack-yr) & $18.3 \pm 23.4$ & $40.8 \pm 28.8$ & $42.8 \pm 30.2$ & $<0.01^{\mathrm{ab}}$ \\
\hline Smoking status, n (\%) & & & & $<0.001^{\mathrm{ab}}$ \\
\hline Never smoker & $38(52.05 \%)$ & 0 & 0 & - \\
\hline Current smoker & $20(27.40 \%)$ & $48(71.64 \%)$ & $17(53.10 \%)$ & $<0.001$ \\
\hline Ex-smoker & $15(20.55 \%)$ & 19 (28.36\%) & $15(46.90 \%)$ & $<0.001$ \\
\hline \multicolumn{5}{|l|}{ Occupation, n (\%) } \\
\hline Manual work in industry & $14(19.18 \%)$ & $12(17.91 \%)$ & $3(9.38 \%)$ & \multirow[t]{5}{*}{0.809} \\
\hline Manual work in service & $10(13.70 \%)$ & $11(16.42 \%)$ & $3(9.38 \%)$ & \\
\hline Farmer & $40(54.79 \%)$ & $33(49.25 \%)$ & $20(62.50 \%)$ & \\
\hline Professionals & $4(5.48 \%)$ & $7(10.45 \%)$ & $4(12.50 \%)$ & \\
\hline Others & $5(6.85 \%)$ & $4(5.97 \%)$ & $2(6.24 \%)$ & \\
\hline CAT score & - & $8(3,11)$ & $18(14,24)$ & $<0.001^{c}$ \\
\hline MMRC score & - & $1(0,1)$ & $3(2,3)$ & $<0.001^{c}$ \\
\hline $\mathrm{FEV}_{1}(\mathrm{~L})$ & $2.80 \pm 0.56$ & $2.07 \pm 0.52$ & $0.96 \pm 0.34$ & $<0.001^{\mathrm{abc}}$ \\
\hline $\mathrm{FEV}_{1} \%$ & $95.07 \pm 14.53$ & $73.88 \pm 16.72$ & $34.35 \pm 9.91$ & $<0.001^{\mathrm{abc}}$ \\
\hline $\mathrm{FVC}(\mathrm{L})$ & $3.66 \pm 0.68$ & $3.44 \pm 0.65$ & $2.31 \pm 0.53$ & $<0.001^{b c}$ \\
\hline FVC\% & $99.73 \pm 13.90$ & $97.13 \pm 15.44$ & $66.53 \pm 12.63$ & $<0.001^{b c}$ \\
\hline $\mathrm{FEV}_{1} / \mathrm{FVC} \%$ & $76.22 \pm 6.32$ & $60.09 \pm 8.64$ & $42.82 \pm 10.95$ & $<0.001^{\mathrm{abc}}$ \\
\hline
\end{tabular}

Current smokers were defined as those who smoked more than five cigarettes per day during the past month; ex-smokers were defined as those who had smoked more than five cigarettes previously and had stopped for > 1 month; nonsmokers were defined as those who had never smoked or patients who smoked no more than five cigarettes per day at the time of the or previously. Data are shown as means \pm SD or median (interquartile range). Differences in continuous variables were evaluated using ANOVA, and $p$ values were corrected using the Bonferroni method

FEV $_{1}$, forced expiratory volume in $1 \mathrm{~s} ;$ FVC forced vital capacity, BMI body mass index, NS no significant difference, CAT COPD assessment test, MMRC modified Medical Research Council

${ }^{a} p<0.05$ for equality between the chronic obstructive pulmonary disease (COPD) I-II group and healthy subjects

${ }^{\mathrm{b}} p<0.05$ for equality between the COPD III-IV group and healthy subjects

${ }^{c} p<0.05$ for equality between the COPD III-IV and COPD I-II groups.

Bacteroidetes ( $p=0.040$ vs. healthy group; $p=0.007$ vs. COPD I-II group) but a higher abundance of Firmicutes $(p=0.041$ vs. COPD I-II group; Fig. $2 \mathrm{~b}$ and Additional file 4: Table S1). The sequences represented in the four phyla were mainly distributed into 15 bacterial families, which accounted for over $95 \%$ of the total sequences in all three groups. Three bacterial families differed in their relative abundance in samples from the three groups (Fig. 2c-e and Additional file 4: Table S2). A higher abundance of Prevotellaceae was observed in the COPD I-II group ( $p=0.046$ vs. healthy controls). Compared with the healthy controls, COPD III-IV subjects exhibited a lower abundance of Bacteroidaceae $(p=0.034)$ and Fusobacteriaceae $(p=0.002)$. There were no significant differences between COPD I-II and COPD III-IV subjects.

In $\beta$-diversity analysis, the variance of the 172 samples was largely explained by the first principal component (54.9\%), corresponding to differing enterotypes but not clinical groups (Fig. 3a). Most healthy controls (68.5\%) were clustered in the 'Bacteroides' enterotype (ET_B), while approximately $45 \%$ of subjects in each COPD patient group were clustered in the 'Prevotella' enterotype ( $\mathrm{X}^{2}$ test, $p=0.072$, healthy vs. COPD I-II subjects; $p=0.149$, healthy vs. COPD III-IV subjects; $p=0.091$, healthy vs. all COPD subjects; Fig. 3b). Permutational multivariate analysis of variance of Bray-Curtis dissimilarity demonstrated statistically significant differences in gut microbiota composition between healthy controls and all COPD subjects $(p=0.011)$.

\section{SCFA profiles}

We compared the levels of intestinal SCFAs in stool samples from 60 healthy controls, 63 COPD I-II patients, and 30 COPD III-IV patients by gas chromatography. All seven SCFAs were detected in all stool samples. Acetic, propionic, and butyric acids accounted for over $89 \%$ of total SCFAs in all three groups, with acetic acid being most abundant (37.4-41.3\%). Total SCFAs levels were significantly lower in the COPD III-IV group than in healthy $(p=0.012)$ and COPD I-II $(p=0.109)$ subjects (Fig. 4a). Moreover, a significant decrease in acetic acid levels was observed in the stool samples of COPD 


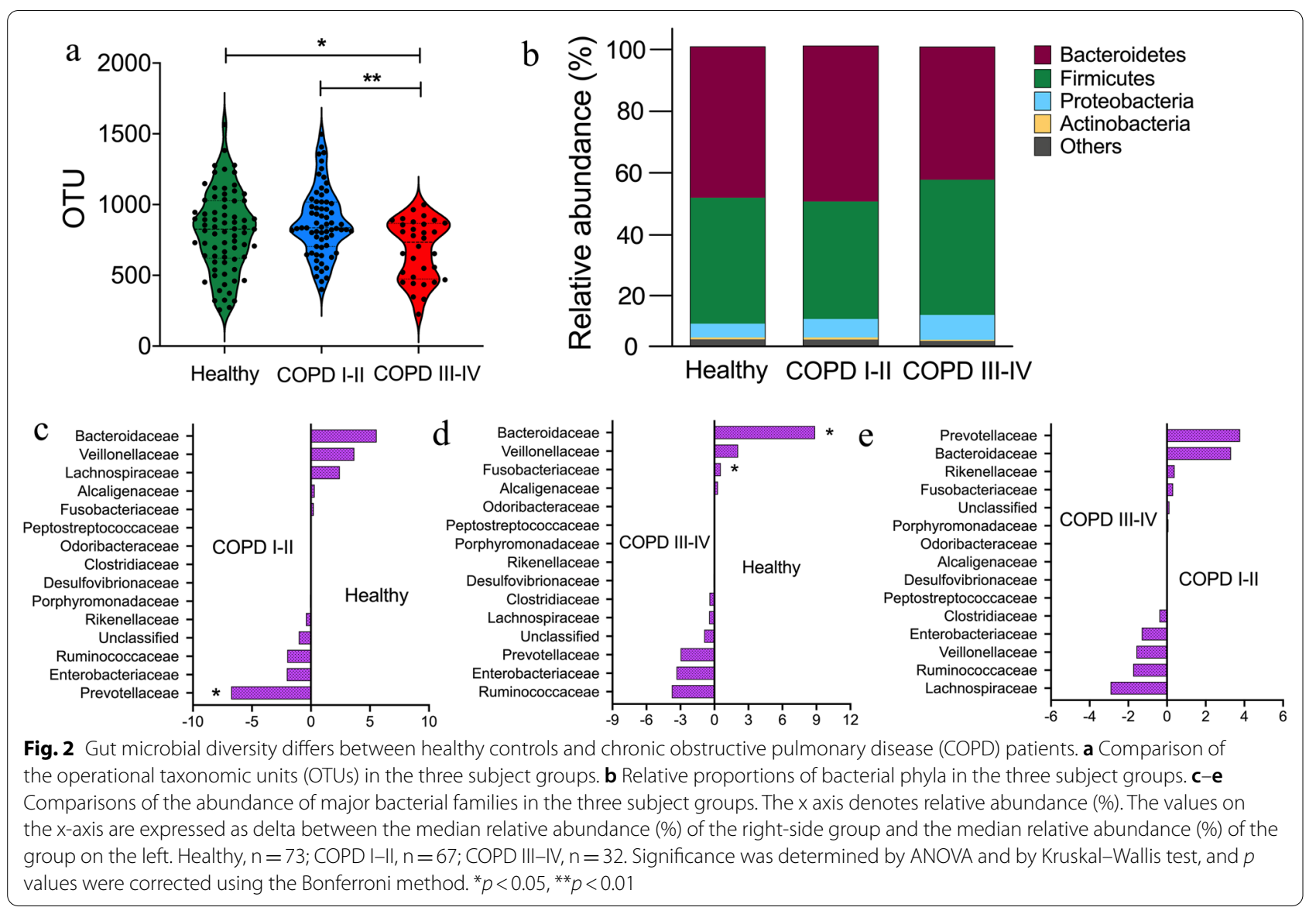

III-IV subjects ( $p=0.007$, Fig. $4 \mathrm{~b})$. Levels of isobutyric acid ( $p=0.016$ vs. healthy group; $p=0.007$ vs. COPD III group) and isovaleric acid ( $p=0.038$ vs. healthy group; $p=0.006$ vs. COPD I-II group) were also significantly lower in the COPD III-IV subjects (Fig. 4c, d). There was no significant difference in the levels of propionic acid, butyric acid, valeric acid, or caproic acid between groups (Fig. 4e-h). Overall, the severity of COPD was correlated with reductions in the levels of SCFAs.

\section{Intestinal microbiota from COPD patients induced lung inflammation and adaptive immune responses in mouse model}

After a 3-week antibiotics treatment, the gut bacterial abundance from mice was significantly lower (Fig. 5a-c). Compared with PBS FMT group, the mice that received human fecal transplants over 4 weeks exhibited higher bacterial diversity (Fig. 5d-f) and differed microbiota composition (Additional file 1: Fig. S1, Additional file 4: Table S2), confirming successful gut microbiota transfer. The viability of bacteria in the inoculum can be seen in Additional file 4: Table S3. A significant reduction in body weight was observed in mice that received microbiota from COPD III-IV patients $(p<0.01$ vs. healthy controls, Fig. 6a) on day 28. Increased plasma cytokine levels were observed in the mouse recipients of fecal microbiota from COPD III-IV patients: levels of interleukin (IL) $1 \beta(p=0.034)$ and tumor necrosis factor $\alpha(p=0.029)$ were significantly higher (Fig. 6b), and an upward trend was observed for IL-6, IL-10, IL-17A, and interferon $\gamma$, although these changes were not statistically significant. Mouse recipients of fecal microbiota from COPD III to IV subjects had higher total cell counts and the percentage of leukocytes in bronchoalveolar lavage fluid ( $p<0.05$, Fig. $6 c, d)$, when compare to the healthy FMT group. Flow cytometry immunophenotyping was performed on T cells and B cells in whole blood. $\mathrm{T}$ and $\mathrm{B}$ lymphocyte sub-populations were defined as $\mathrm{B}$ lymphocytes (CD3 - CD19+cells), auxiliary $\mathrm{T}$ lymphocytes (CD3+CD4+ cells), and cytotoxic T lymphocytes (CD3 + CD8 + cells). CD3 $+\mathrm{T}$ lymphocyte counts were higher in the peripheral blood of mice that received fecal microbiota from COPD I to II patients $(p<0.01)$ and COPD III-IV patients $(p<0.01)$ than from healthy controls (Fig. 6e). Increased counts of auxiliary $\mathrm{T}$ lymphocytes $(p<0.01$; Fig. 6f) and cytotoxic T lymphocytes 
A

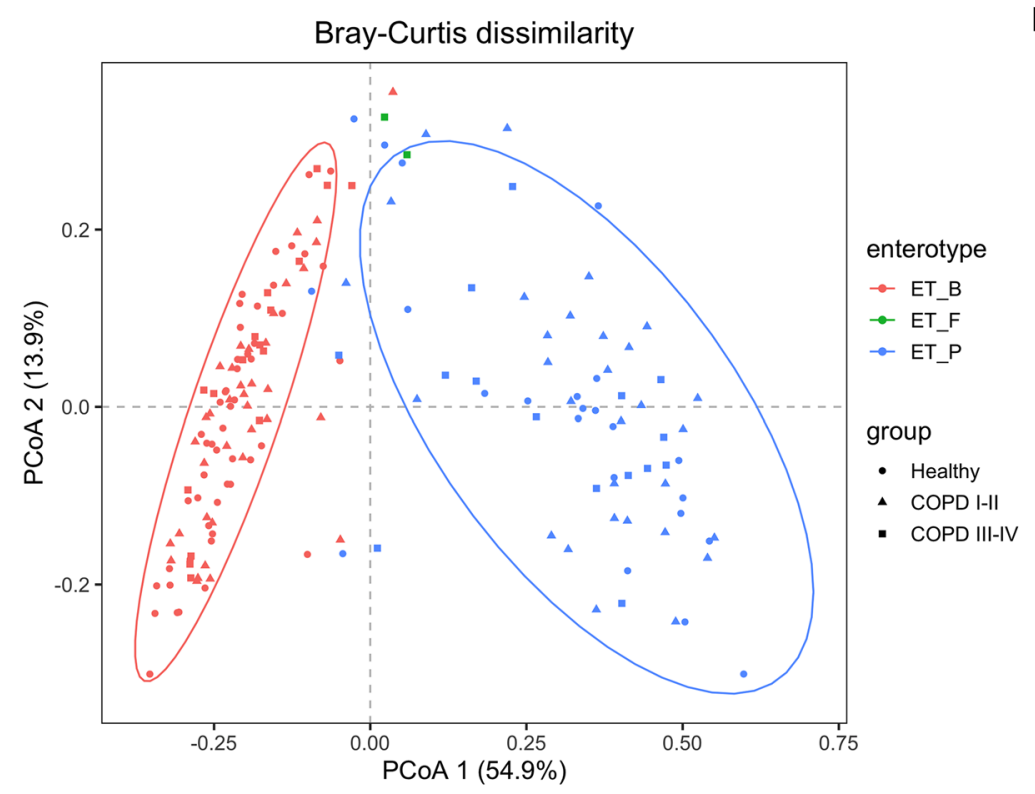

B

Reference-based enterotypes

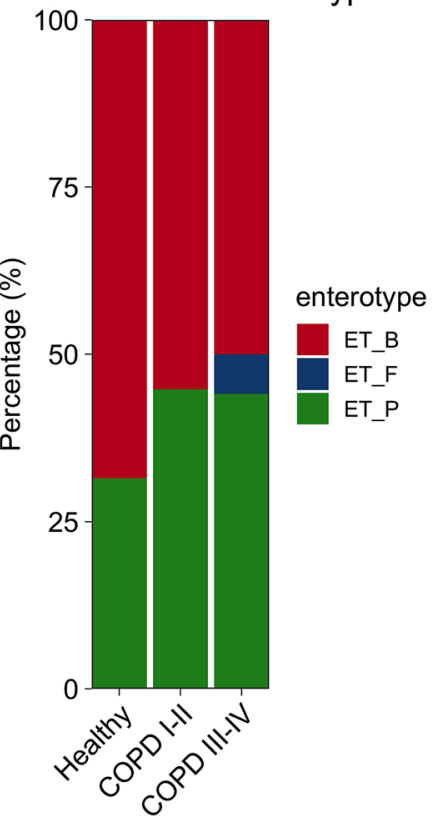

Fig. 3 Gut enterotypes shift in human adults with chronic obstructive pulmonary disease (COPD). a Enterotypes in clinical groups. A total of 172 samples (Healthy, $n=73$; COPD I-II, $n=67$; COPD III-IV, $n=32$ ) are clustered into ET_P ('Prevotella' enterotype, blue) and ET_B ('Bacteroides' enterotype, red) by PCoA of Bray-Curtis dissimilarity at the genus level. b Percentage of individuals from each group characterized by ET_P (green), ET_B (red), and ET_F ('Firmicutes' enterotypes, blue). The major contributors in the two enterotypes are Prevotella and Bacteroides, respectively. Each dot corresponds to a sample

$(p<0.01$; Fig. 6g) and decreased counts of B lymphocytes $(p<0.01$; Fig. 6h) were observed in mice that received fecal microbiota from either COPD group. Overall, a higher proportion and number of $\mathrm{T}$ lymphocytes and lower proportion and number of B lymphocytes were observed in recipient mice that received fecal microbiota from COPD patients.

We also assayed three key proteins to assess the activation of airway remodeling and mucus hypersecretion in the lung. Higher levels of $\alpha$ smooth-muscle actin were observed in the COPD III-IV FMT mouse group $(p<0.01$, Fig. 7a) and expression of matrix metalloproteinase 2 around airways was higher in both COPD FMT mouse groups $(p<0.05$ or 0.01 , Fig. $7 \mathrm{~b})$. Immunohistochemistry showed that intestinal microbiota from patients with COPD III-IV promoted mucus hypersecretion (AB-PAS and MUC5AC, $p<0.01$, Fig. 7c, d) in recipient mice. These results show that gut microbiota from COPD patients induced a greater pulmonary inflammatory response than microbiota from healthy controls.

\section{Intestinal microbiota accelerates COPD development in mice}

To further assess whether gut microbiota dysbiosis is a potential causal factor in the progression of COPD, we performed fecal microbiota transplantation in mice during biomass smoke exposure over 20 weeks. Additional file 3: Fig. S3 show the inhalation chamber and the size distributions of particulates inhaled by the mice during exposure. Additional file 4: Table S4 lists the concentrations of oxygen, carbon monoxide, nitrogen oxides, and sulfur dioxide during the exposures. After 20 weeks of exposure, the PEF, FEV ${ }_{20}$, MMEF and MV were significantly lower in the mouse recipients of fecal microbiota from COPD I-II and III-IV patients, while Cchord and FRC were significantly higher $(p<0.05, p<0.01$ or 0.001 , Fig. 8a-f). Histological analysis showed that the mean linear intercept was significantly larger in mice that received fecal microbiota from COPD patients than in mice from the PBS and healthy controls groups $(p<0.01$, Fig. 9a). Bronchial walls were thicker in the lungs of mice from the COPD III-IV FMT group $(p<0.05$, Fig. 9b). Accelerated declines in lung function and emphysematous changes were observed in the mouse recipients of fecal microbiota from COPD patients during biomass smoke exposure.

After 20 weeks of biomass smoke exposure, higher levels of the activation of airway remodeling and mucus hypersecretion-associated proteins were observed in mice that received fecal microbiota from 


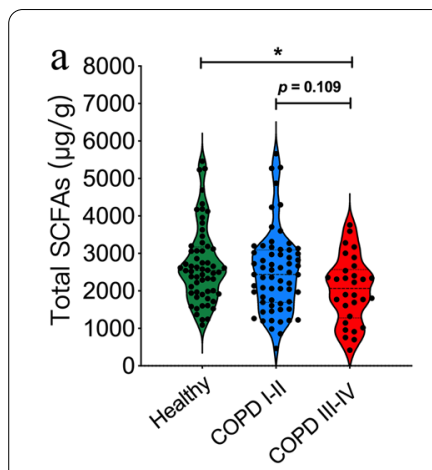

$\mathrm{e}$
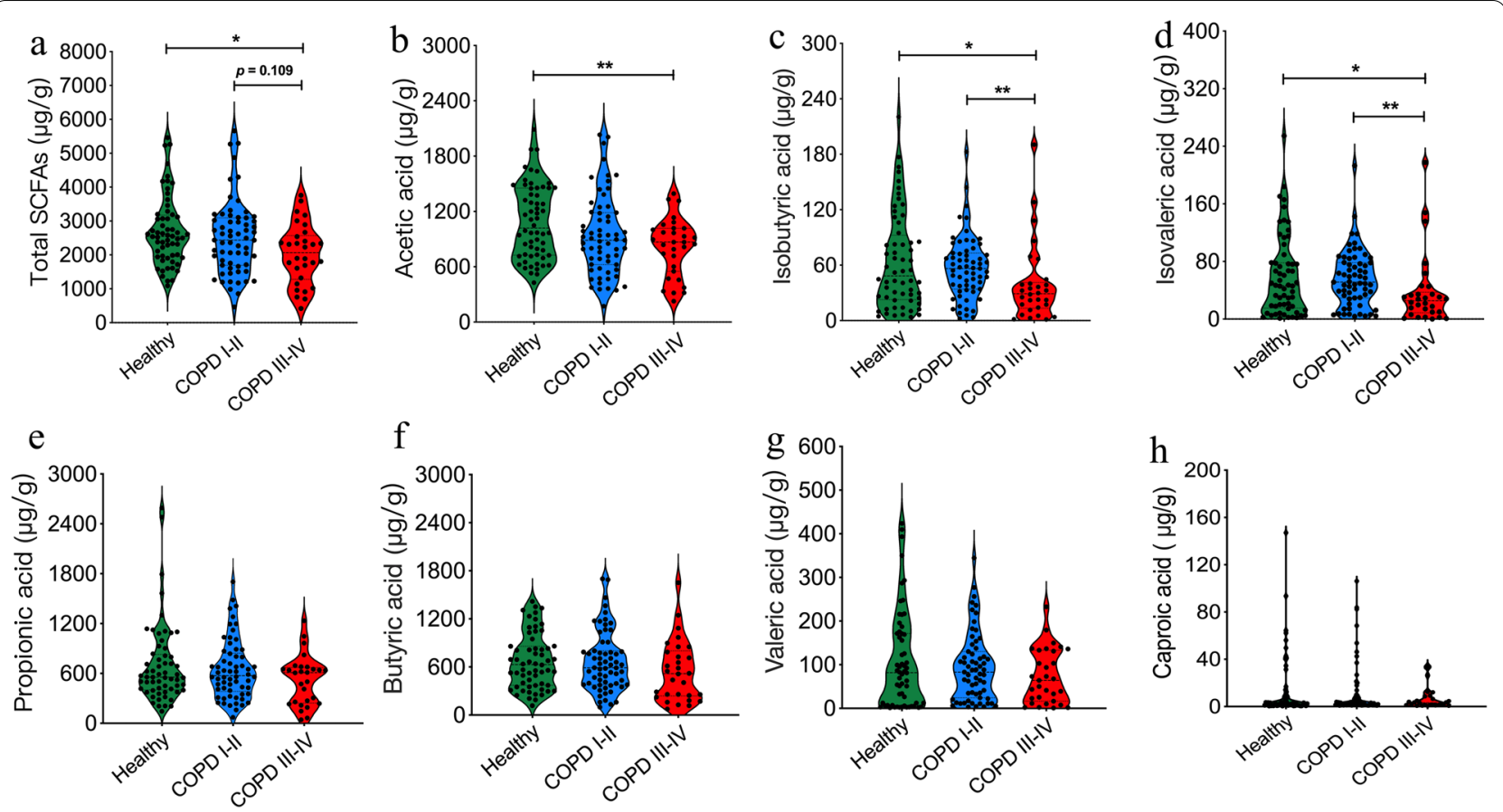

Fig. 4 Short-chain fatty acid levels in fecal samples from healthy, chronic obstructive pulmonary disease (COPD) I-II subjects, and COPD III-IV subjects. a The levels of total short-chain fatty acids were significantly lower in COPD III-IV subjects. b-d Acetic acid, isobutyric acid, and isovaleric acid levels were also lower in COPD III-IV subjects. e-h Reductions in the levels of propionic acid, butyric acid, valeric acid, and caproic acid were observed in COPD patients, although this trend was not statistically significant. Significance was determined by the ANOVA test, and $p$ values were corrected using the Bonferroni method. Each dot corresponds to a sample. Healthy, $n=60 ;$ COPD I-II, $n=63 ;$ COPD III-IV, $n=30 .{ }^{*} p<0.05,{ }^{* *} p<0.01$

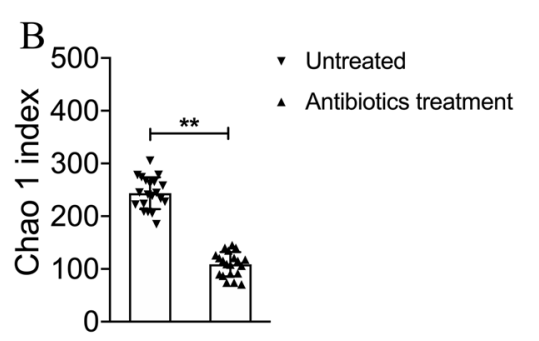

$\mathrm{D}$

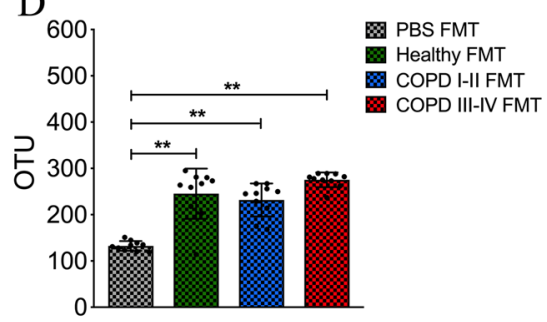

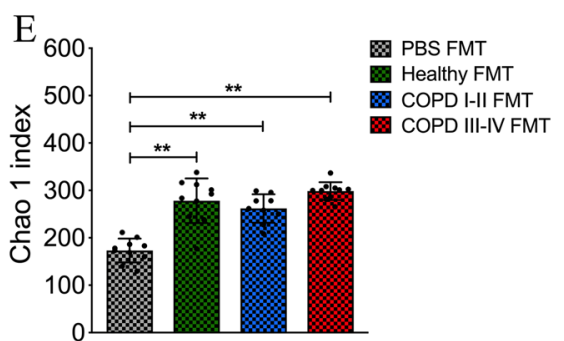

Fig. 5 Microbial abundance and diversity in mice following antibiotics treatment and transplants of human fecal matter. a-c The gut bacterial abundance (operational taxonomic units, OTUs) and diversity (Chao1 index and Shannon index) in mice was significantly lower after treatment with broad-spectrum antibiotics in drinking water for 3 weeks, $n=20$ mice. $\mathbf{d}-\mathbf{f}$ The mice that received human fecal transplants over 28 days exhibited higher gut bacterial abundance and diversity than the PBS FMT group (no fecal transplants), $n=10$ mice. ${ }^{*} p<0.05,{ }^{* *} p<0.01$ 

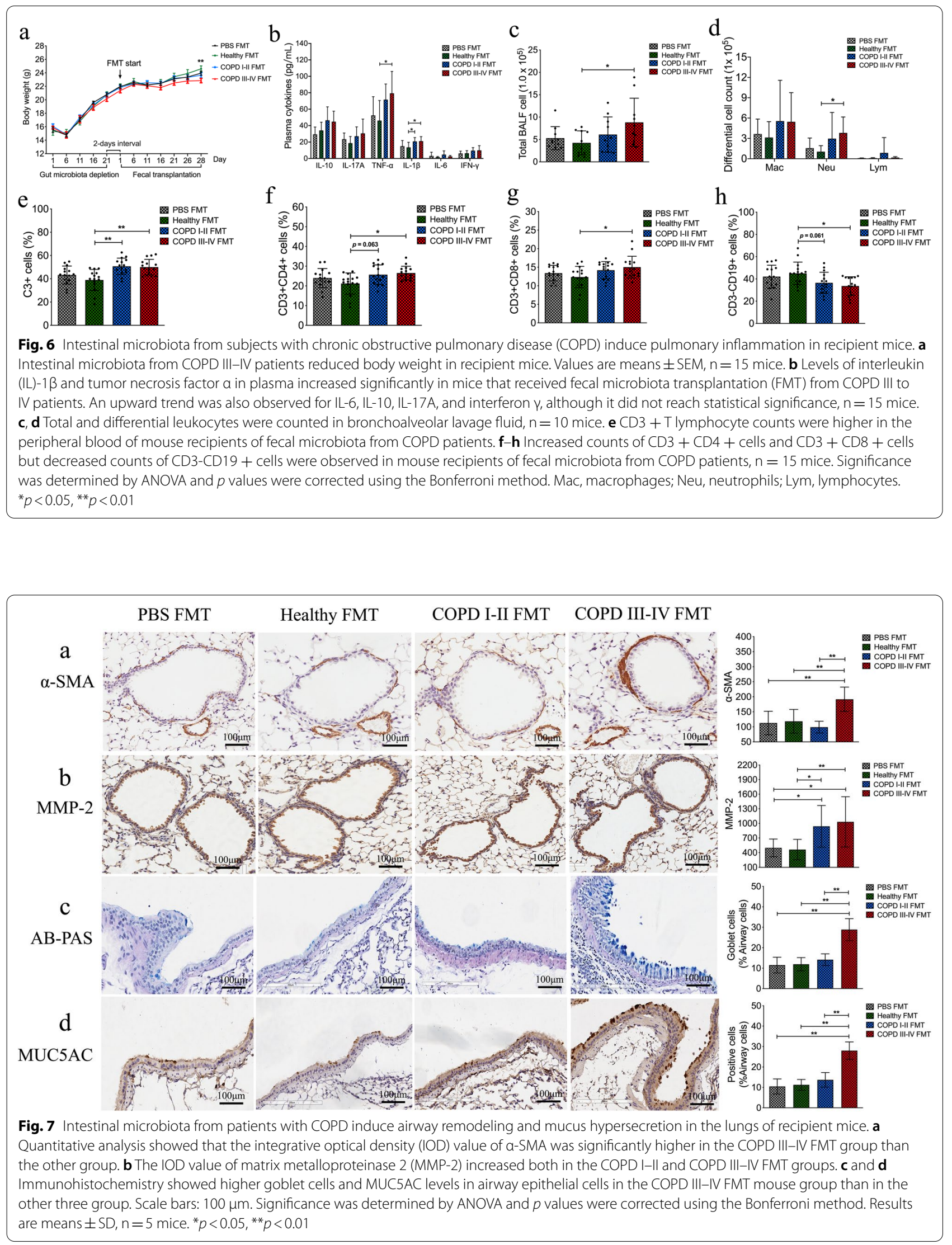

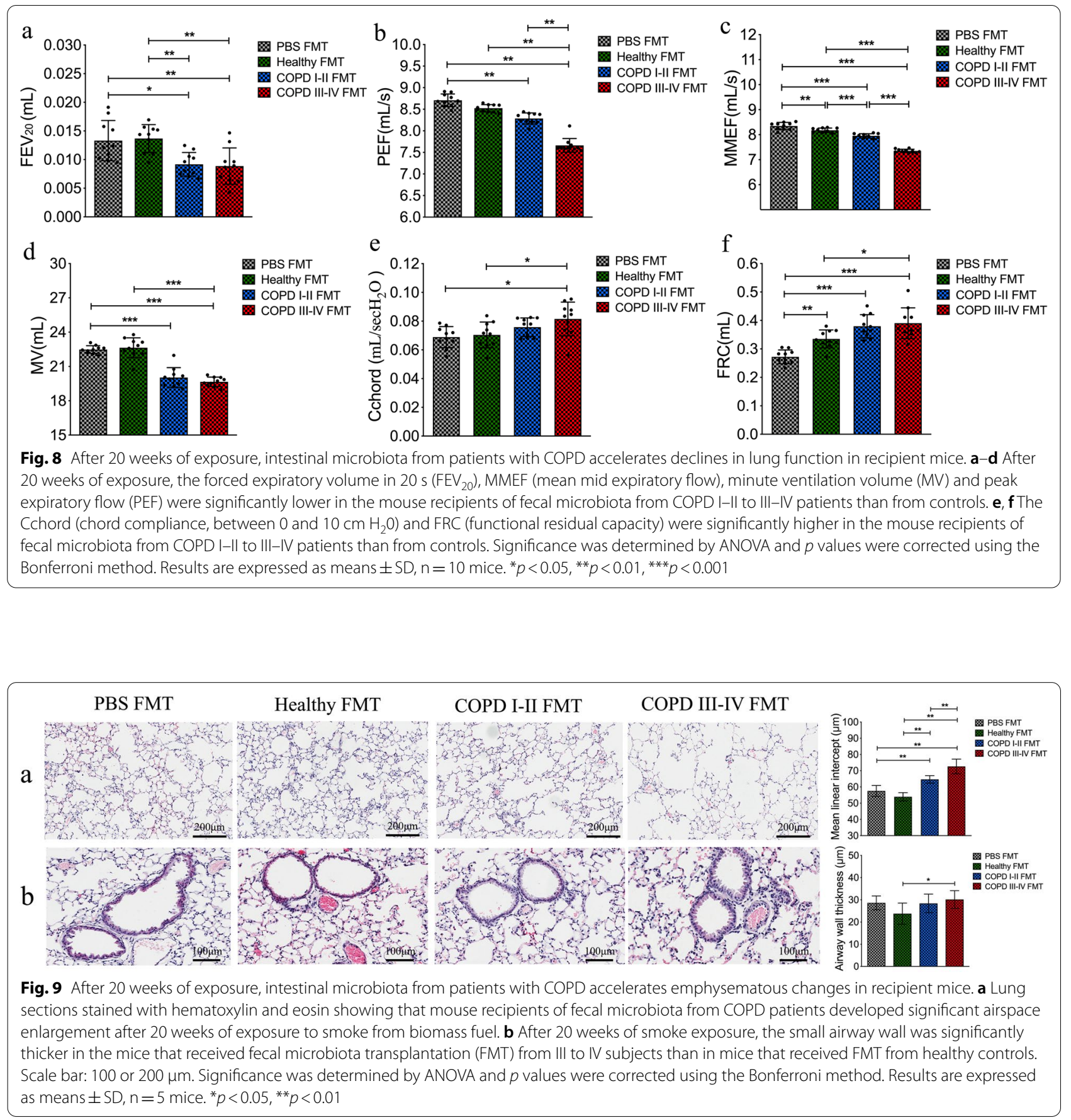

COPD patients. Higher levels of claudin 1, $\alpha$-SMA, NE and MMP-2 were observed in the COPD III-IV FMT mouse group ( $p<0.01$ or 0.01 , Fig. 10a). Furthermore, higher levels of MUC5AC were observed in the COPD III-IV FMT mouse group and expression of MUC2 was higher in both COPD FMT mouse groups $(p<0.01$ or 0.01 , Fig. 10b).

\section{Discussion}

To date, only a few studies have reported a direct association between the gut microbiome and COPD, and epidemiological evidence is lacking $[31,32]$. We found that the gut microbiome of COPD patients was characterized by a distinct overall microbial diversity and composition and lower levels of SCFAs, especially in patients with lower 


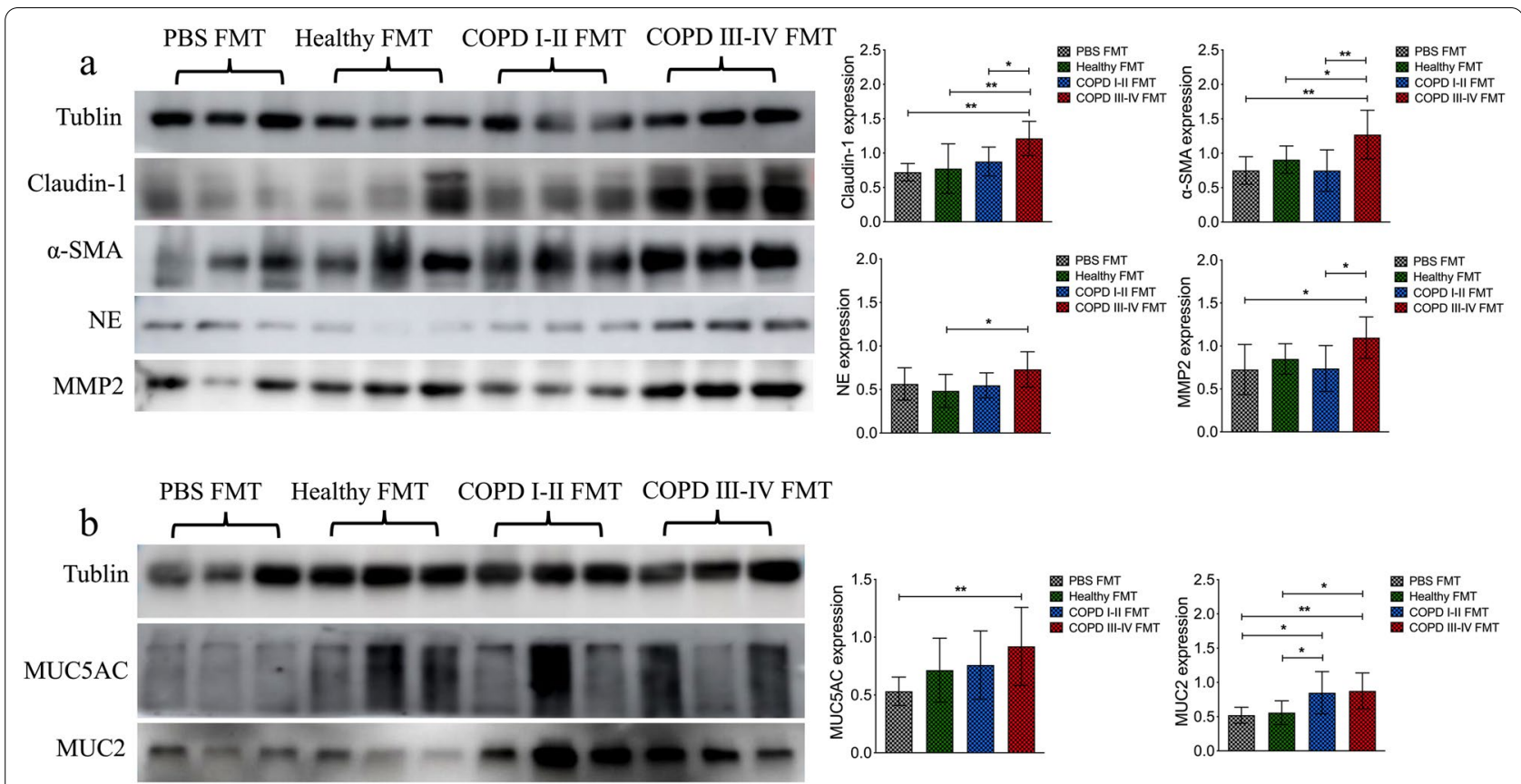

Fig. 10 After 20 weeks of exposure, intestinal microbiota from patients with COPD induced airway remodeling and mucus hypersecretion in recipient mice. a Western blots of lung sections show that the expression levels of claudin 1, a smooth-muscle actin (a-SMA), neutrophil elastase (NE), and matrix metalloproteinase 2 (MMP-2) increased markedly in the COPD III-IV group in comparison with the healthy controls and COPD I-II fecal microbiota transplantation mice. b Representative western blots showing increased MUC5AC in the lungs of mice that received fecal microbiota transplantation from COPD III-IV patients. Higher levels of MUC2 were observed in both COPD FMT mouse groups. Significance was determined by ANOVA, and $p$ values were corrected using the Bonferroni method. Results are expressed as mean $\pm S D, n=10$ mice. ${ }^{*} p<0.05$, ${ }^{* *} p<0.01$

pulmonary function (COPD III-IV). As the subjects were from a relatively restricted environment, the differences in their diets were small, limiting variations in the gut microbiome [33]. Our work provides the first direct evidence that altered gut microbiota in COPD patients is associated with airway inflammation and accelerated COPD progression in mice.

Dysbiosis in the gut microbiota has been linked to alterations in immune responses and to the development of lung diseases such as acute respiratory distress syndrome, pneumonia, and childhood asthma [34-36]. Recent research results show that faecal microbiome and metabolome of COPD patients are distinct from those of healthy individuals [31]. Another study found that gut microbiota composition significantly affects cigarette smoke-induced mouse COPD development, and a commensal bacterium Parabacteroides goldsteinii was shown to ameliorate COPD [32]. Our mouse experiments offer further support for a viewpoint of pathogenic links between microbiota and the gut-lung axis in COPD. We have confirmed the gut microbiota composition in COPD, when transplanted, causes airway inflammation. Furthermore, intestinal microbiota from COPD patients induced mucus hypersecretion and accelerated lung function decline and emphysematous changes in mouse lungs.

Chronic sterile inflammation is central to the pathobiology of obesity and obesity-related complications $[37,38]$, and previous research supports dysbiosis of Bacteroidetes and Firmicutes as a potential trigger of inflammation [39, 40]. Our results found that the relative proportion of Bacteroidetes was lower and that of Firmicutes was higher in COPD group when compare to healthy control, consistent with reports from studies of obesity and diabetes. At the family level, we observed differing relative abundances between groups for Fusobacteriaceae, Prevotellaceae, and Bacteroidaceae. Prevotella were enriched in the stool of COPD patients, which were previously identified as highly enriched in the gut microbiota of patients with rheumatoid arthritis [41]. Colonization with Prevotella was shown to exacerbate epithelial inflammation and reduce body weight in mice [42]; we observed a similar reduction in body weight and higher pulmonary inflammation in mice colonized with gut microbiota from COPD III to IV patients. Thus, Prevotella may be involved in COPD, perhaps by triggering the inflammatory response somehow. Individual bacterial species should be monocolonized in mice for 
a systematic analysis of host lung pathology/immune responses to colonization, further individual bacterial transfer experiments may be beneficial to clarify the mechanisms underlying the effect of gut microbiota in COPD pathogenesis.

In this study, the levels of SCFAs in stool from COPD III-IV patients were significantly lower than in stool from the other groups. This seems to be consistent with our previous research, that is gut microbial dysbiosis and lower levels of short-chain fatty acids were observed in a particulate matter-induced rat COPD model [27]. SCFAs have anti-inflammatory properties, improve gut barrier function and reduce intestinal bacterial translocation in the host. SCFAs are associated with reduced risk of various conditions, including COPD, irritable bowel disease, allergic asthma, and diabetes [43-46]. The potential of SCFAs to protect against lung inflammation and emphysema has been demonstrated in animal models [47, 48]. Hence, increasing SCFAs intake in COPD may confer a positive effect, as previously suggested [43].

The gut microbiota composition in COPD, when transplanted, causes COPD-like systemic and airway inflammation. $\mathrm{CD} 3+\mathrm{CD} 4+$ and $\mathrm{CD} 3+\mathrm{CD} 8+\mathrm{T}$ cells were present in higher proportions in both COPD mouse groups. A growing body of evidence suggests that adaptive immune reactions are involved in the pathogenesis of COPD, especially abnormalities in the number and function of CD4+ and CD8 + T cell $[49,50]$. Airway inflammation is a feature of COPD and is present in both the large and small airways. Our results demonstrate that intestinal microbiota from COPD patients caused lung inflammation and airway mucus hypersecretion in mice. Numerous studies have shown that mucus hypersecretion is a key factor in the development of COPD and could permanently damage the pulmonary function [51]. Furthermore, mucus hypersecretion in the large and small airways is a consistent feature in a murine model of COPD [28]. We also looked at the effects of gut microbiota on airway remodeling-associated proteins, since the link between increased airway remodeling and COPD is well-established [2].

Our study had several limitations. First, we surveyed the gut microbiota from healthy controls and COPD patients cross-sectionally. In the absence of longitudinal or interventional study designs, it is difficult to ascertain whether gut microbiota dysbiosis is a cause or a consequence of COPD. Second, there was significant difference in smoking history between the COPD patients and healthy controls in our cohorts, hence smoking can be a confounding factor in our study. Inclusion of an additional healthy control subgroup with matched smoking status both for gut microbiota profiling and fecal transplantation experiment is needed to better address this problem. Third, the COPD patients enrolled in this study were mixed with cigarette smoke exposure and biofuel exposure which may have an impact on research results. Fourth, the gut microbiota represents a large community of microorganisms, and each bacterial species should be monocolonized in mice for a systematic analysis of host lung pathology/immune responses to colonization. Lastly, in the mouse model of microbiome depletion by administration of antibiotics, lung microbiome will also be reduced along with gut microbiome. How the lung microbiome depletion influences the development of COPD are largely unknow.

\section{Conclusion}

In summary, we present evidence for dysbiosis in the gut microbiota of COPD patients along with reduced levels of SCFAs and establish a potential link between gut microbiota dysbiosis, airway inflammation, and COPD progression. The role of the gut microbiome in the development of COPD should draw considerable attention.

\section{Abbreviations}

COPD: Chronic obstructive pulmonary disease; IL: Interleukin; PBS: Phosphatebuffered saline; FMT: Fecal microbiota transplantation; SCFA: Short-chain fatty acid.

\section{Supplementary Information}

The online version contains supplementary material available at https://doi. org/10.1186/s12931-021-01872-z.

\begin{abstract}
Additional file 1: Fig. S1. Phylum percent relative abundances. To determine the response of the host microbiome after receiving human fecal transplants, we analyzed the taxonomical community structure of the microbiome in mice fecal samples from the PBS FMT group, healthy FMT group, COPD I-II FMT group, and COPD III-IV FMT group. At the phylum level, all samples from the PBS FMT group, healthy FMT group, COPD I-II FMT group, and COPD III-IV FMT group contained four major bacterial phyla (\%): Bacteroidetes, 36.84 (26.06, 45.97), 32.13 (23.29, 35.10), $33.05(19.60,37.07)$, and $29.77(23.90,32.58)$, respectively; Firmicutes, 40.33 $(26.12,50.17), 49.88(44.50,53.56), 53.60(47.35,68.73)$, and $54.26(45.75$, 57.32), respectively; Proteobacteria, $8.51(5.20,13.30), 3.30(1.74,7.29), 1.04$ $(0.49,5.23)$, and $3.24(1.06,8.83)$, respectively; Actinobacteria, $0.06(0.04$, $1.42), 9.97(3.33,11.77), 7.11(4.52,10.32)$, and $4.49(2.98,8.92)$, respectively. Relative abundances of bacterial phyla differed ( $p<0.05$ or $p<0.01$ ) between the PBS FMT group and fecal transplants group in Actinobacteria, Firmicutes, and Proteobacteria. There was no significant difference between the healthy FMT group, COPD I-II FMT group, and COPD III-IV FMT group. Results are expressed as the mean; $n=10$ mice. Significance was determined by ANOVA and by Kruskal-Wallis test, and $p$ values were corrected using the Bonferroni method.
\end{abstract}

Additional file 2: Fig. S2. Microbial abundance was measured in feces from human subjects and compared with microbial abundance in feces from recipient mice. At the phylum level, inoculum from healthy subjects, COPD I-II subjects, and COPD III-IV subjects contained four major bacterial phyla (\%): Bacteroidetes, 54.50, 51.48 and 43.43, respectively; Firmicutes, 38.50, 39.04 and 46.96, respectively; Proteobacteria, 3.67, 6.22 and 7.53, respectively; Actinobacteria, 0.69, 1.08 and 0.65, respectively. As shown below, the characteristics of the murine gut microbial community 
post-fecal transplant were not identical to those profiled originally in the human-derived sample $(\mathrm{A}-\mathrm{C})$. Results are expressed as the mean.

Additional file 3: Fig. S3. The biomass smoke exposure system and particle size distributions during exposure. (a) All mice were exposed to biomass smoke in inhalation chamber systems. (b) Particulate matter concentrations and particle size distributions during biomass smoke exposure. Boxes and the inside line represent means \pm SD for particulate matter with an aerodynamic diameter $<2.5 \mu \mathrm{m}$ (PM2.5).

Additional file 4: Supplementary table 1-4. Supplementary table 1. Relative abundance of most abundant phyla (\%) observed in stool samples collected in healthy control group and COPD group. Supplementary table 2. Relative abundance of most abundant family (\%) observed in stool samples collected in healthy control group and COPD group. Supplementary table 3 . The viability of bacteria in the inoculum in different microbial growth medium (CFU/ml). Supplementary table 4. Concentrations of particulate matter (PM) and gaseous pollutants measured during exposure.

Additional file 5: Ethics approval and consent to participate.

Additional file 6: Animal ethics approval.

\section{Acknowledgements}

We thank Liwen Bianji, Edanz Group China (www.liwenbianji.cn/ac), for editing the English text of a draft of this manuscript.

\section{Authors' contributions}

NJL, ZLD, PXR, JL, YMZ, and BL conceived the study, directed the project, and designed the experiments; NJL and ZLD obtained the samples and clinical information, interpreted the results, and wrote the manuscript; NJL, ZW, and ZWY performed the microbial sequencing analysis; THP, JHZ, WTC, ZSD, and JDP performed the fecal microbiota transplantation and analyzed the animal data; all authors read and approved the final manuscript.

\section{Funding}

This work was supported by a grant from the National Natural Science Foundation of China (81970045 and 81900030), Local Innovative and Research Teams Project of Guangdong Pearl River Talents Program (2017BT01S155), Guangdong Province Key Field R\&D Program (2020B1111330001), Youth Foundation of the National Key Laboratory of Respiratory Diseases (SKLRDQN-201908) and Science and Technology Program of Guangzhou.

\section{Availability of data and materials}

The raw sequencing data were deposited in the Sequence Read Archive database under accession number PRJNA606975. The R codes and detailed instruction for the enterotype analysis in this manuscript are now provided in GitHub (https://github.com/yzhaowei/2020_COPD_enterotypes/).

\section{Declarations}

\section{Ethics approval and consent to participate}

The Ethics Commission of the First Affiliated Hospital of Guangzhou Medical University approved the study (No.2017-21, Additional file 5: e-Appendix 1). Volunteers provided voluntary, written informed consent before taking part in study procedures. All animal care and experimental protocols complied with the guiding principles for the care and use of laboratory animals recommended by the Chinese Association for Laboratory Animal Science Policy. The Animal Medical Center of Guangzhou Medical University reviewed and approved all experiments (identification number: GY2018-084, Additional file 6: e-Appendix 2).

\section{Consent for publication}

Not applicable.

\section{Competing interests}

There are no conflicts of interest to declare. There is also no non-author involvement in the preparation of the manuscript.

\section{Author details}

${ }^{1}$ Department of Allergy and Clinical Immunology, State Key Laboratory of Respiratory Disease, National Clinical Research Center for Respiratory Disease, Guangzhou Institute of Respiratory Health, The First Affiliated Hospital of Guangzhou Medical University, 151 Yanjiang Road, Guangzhou, Guangdong, People's Republic of China. ${ }^{2}$ College of Medicine, Lishui University, Lishui, Zhejiang, People's Republic of China. ${ }^{3}$ Institute of Ecological Science, School of Life Science, South China Normal University, Guangzhou, Guangdong, People's Republic of China. ${ }^{4}$ The GMU-GIBH Joint School of Life Sciences, Guangzhou Medical University, Guangzhou, Guangdong, People's Republic of China.

Received: 30 May 2021 Accepted: 18 October 2021

Published online: 25 October 2021

\section{References}

1. Wang $C, X u J$, Yang $L$, et al. Prevalence and risk factors of chronic obstructive pulmonary disease in China (the China Pulmonary Health [CPH] study): a national cross-sectional study. Lancet. 2018;391:1706-17.

2. Rabe KF, Watz H. Chronic obstructive pulmonary disease. Lancet. 2017;389(10082):1931-40.

3. $2021 \mathrm{Global}$ strategy for prevention, diagnosis and management of COPD. https://goldcopd.org/2021-gold-reports/.

4. Vestbo J, Anderson JA, Brook RD, SUMMIT Investigators, et al. Fluticasone furoate and vilanterol and survival in chronic obstructive pulmonary disease with heightened cardiovascular risk (SUMMIT): a double-blind randomised controlled trial. Lancet. 2016;387(10030):1817-26.

5. Hurst JR, Sin DD. Chronic obstructive pulmonary disease as a risk factor for cardiovascular disease. A view from the SUMMIT. Am J Respir Crit Care Med. 2018;198(1):2-4.

6. Parpaleix A, Boyer L, Wiedemann A, et al. Impaired humoral and cellular immune responses to influenza vaccination in chronic obstructive pulmonary disease patients. J Allergy Clin Immunol. 2017;140(6):1754-1757. e6.

7. Rutten EPA, Lenaerts K, Buurman WA, et al. Disturbed intestinal integrity in patients with COPD: effects of activities of daily living. Chest. 2014;145:245-52.

8. Durack J, Lynch SV. The gut microbiome: Relationships with disease and opportunities for therapy. J Exp Med. 2019;216(1):20-40.

9. Nie P, Li Z, Wang Y, et al. Gut microbiome interventions in human health and diseases. Med Res Rev. 2019;39(6):2286-313.

10. Li J, Zhao F, Wang Y, et al. Gut microbiota dysbiosis contributes to the development of hypertension. Microbiome. 2017;5(1):14.

11. Bartolomaeus H, Balogh A, Yakoub M, et al. Short-chain fatty acid propionate protects from hypertensive cardiovascular damage. Circulation. 2019;139(11):1407-21.

12. Park HK, Choi Y, Lee DH, et al. Altered gut microbiota by azithromycin attenuates airway inflammation in allergic asthma. J Allergy Clin Immunol. 2020;145(5):1466-9.

13. Keely S, Talley NJ, Hansbro PM. Pulmonary-intestinal cross-talk in mucosal inflammatory disease. Mucosal Immunol. 2012;5:7-18.

14. Budden KF, Gellatly SL, Wood DL, et al. Emerging pathogenic links between microbiota and the gut-lung axis. Nat Rev Microbiol. 2017;15(1):55-63.

15. Chakradhar S. A curious connection: teasing apart the link between gut microbes and lung disease. Nat Med. 2017;23(4):402-4.

16. Zhou Y, Zhong NS, Li X, et al. Tiotropium in early-stage chronic obstructive pulmonary disease. N Engl J Med. 2017;377(10):923-35.

17. Li C, Zhou Y, Liu S, et al. Tiotropium discontinuation in patients with earlystage COPD: a prospective observational cohort study. ERJ Open Res. 2019;5(1):00175-2018.

18. Liu S, Zhou Y, Liu S, et al. Association between exposure to ambient particulate matter and chronic obstructive pulmonary disease: results from a cross-sectional study in China. Thorax. 2016;0:1-8.

19. Blake MR, Raker JM, Whelan K. Validity and reliability of the Bristol Stool Form Scale in healthy adults and patients with diarrhoea-predominant irritable bowel syndrome. Aliment Pharmacol Ther. 2016;44(7):693-703.

20. 165 Metagenomic Sequencing Library Preparation. San Diego: Illumina. Updated 2014. Retrieved July 15, 2016, from https://www.lllumina.com/. 
21. Klindworth A, Pruesse E, Schweer T, et al. Evaluation of general $16 \mathrm{~S}$ ribosomal RNA gene PCR primers for classical and next-generation sequencing-based diversity studies. Nucleic Acids Res. 2013;41(1):e1.

22. Rocafort M, Noguera-Julian M, Rivera J, et al. Evolution of the gut microbiome following acute HIV-1 infection. Microbiome. 2019;7(1):73.

23. Magoc T, Salzberg SL. FLASH: fast length adjustment of short reads to improve genome assemblies. Bioinformatics. 2011;27(21):2957-63.

24. Costea PI, Hildebrand F, Arumugam M, et al. Enterotypes in the landscape of gut microbial community composition. Nat Microbiol. 2018;3(1):8-16.

25. Schuijt TJ, Lankelma JM, Scicluna BP, et al. The gut microbiota plays a protective role in the host defence against pneumococcal pneumonia. Gut. 2016;65(4):575-83.

26. Papić B, Pate M, Henigman U, et al. New approaches on quantification of Campylobacter jejuni in poultry samples: the use of digital PCR and realtime PCR against the ISO standard plate count method. Front Microbiol. 2017:8:331.

27. Li N, Yang Z, Liao B, et al. Chronic exposure to ambient particulate matter induces gut microbial dysbiosis in a rat COPD model. Respir Res. 2020;21(1):271.

28. He F, Liao B, Pu J, et al. Exposure to ambient particulate matter induced COPD in a rat model and a description of the underlying mechanism. Sci Rep. 2017;31(7):45666.

29. Han W, Dong Z, Dimitropoulou C, Su Y. Hydrogen sulfide ameliorates tobacco smoke-induced oxidative stress and emphysema in mice. Antioxid Redox Signal. 2011;15(8):2121-34.

30. Hsia CC, Hyde DM, Ochs M, et al. An official research policy statement of the American Thoracic Society/European Respiratory Society: standards for quantitative assessment of lung structure. Am J Respir Crit Care Med. 2010;181(4):394-418.

31. Bowerman KL, Rehman SF, Vaughan A, et al. Disease-associated gut microbiome and metabolome changes in patients with chronic obstructive pulmonary disease. Nat Commun. 2020;11(1):5886.

32. Lai HC, Lin TL, Chen TW, et al. Gut microbiota modulates COPD pathogenesis: role of anti-inflammatory Parabacteroides goldsteinii lipopolysaccharide. Gut. 2021;322599.

33. He Y, Wu W, Zheng HM, et al. Regional variation limits applications of healthy gut microbiome reference ranges and disease models. Nat Med. 2018:24(10):1532-5.

34. Dickson RP, Singer BH, Newstead MW, et al. Enrichment of the lung microbiome with gut bacteria in sepsis and the acute respiratory distress syndrome. Nat Microbiol. 2016;1(10):16113.

35. Tamburini S, Clemente JC. Neonatal gut microbiota induces lung immunity against pneumonia. Nat Rev Gastroenterol Hepatol. 2017;14(5):263-4.

36. Stokholm J, Blaser MJ, Thorsen J, et al. Maturation of the gut microbiome and risk of asthma in childhood. Nat Commun. 2018;9(1):141.

37. Cox AJ, West NP, Cripps AW. Obesity, inflammation, and the gut microbiota. Lancet Diabetes Endocrinol. 2015;3(3):207-15.
38. Perry RJ, Camporez JG, Kursawe R, et al. Hepatic acetyl CoA links adipose tissue inflammation to hepatic insulin resistance and type 2 diabetes. Cell. 2015;160(4):745-58.

39. Cani PD, Jordan BF. Gut microbiota-mediated inflammation in obesity: a link with gastrointestinal cancer. Nat Rev Gastroenterol Hepatol. 2018;15(11):671-82

40. Qin J, Li Y, Cai Z, et al. A metagenome-wide association study of gut microbiota in type 2 diabetes. Nature. 2012;490(7418):55-60.

41. Alpizar-Rodriguez D, Lesker TR, Gronow A, et al. Prevotella copri in individuals at risk for rheumatoid arthritis. Ann Rheum Dis. 2019;78(5):590-3.

42. Scher JU, Sczesnak A, Longman RS, et al. Expansion of intestinal Prevotella copri correlates with enhanced susceptibility to arthritis. Elife. 2013;2:e01202.

43. Kaluza J, Harris H, Wallin A, et al. Dietary fiber intake and risk of chronic obstructive pulmonary disease: a prospective cohort study of men. Epidemiology. 2018;29(2):254-60.

44. Gonçalves P, Araújo JR, Di Santo JP. A cross-talk between microbiotaderived short-chain fatty acids and the host mucosal immune system regulates intestinal homeostasis and inflammatory bowel disease. Inflamm Bowel Dis. 2018;24(3):558-72.

45. Arrieta MC, Stiemsma LT, Dimitriu PA, et al. Early infancy microbial and metabolic alterations affect risk of childhood asthma. Sci Transl Med. 2015;7(307):307-152.

46. Lau WL, Vaziri ND. Gut microbial short-chain fatty acids and the risk of diabetes. Nat Rev Nephrol. 2019;15(7):389-90.

47. Tomoda K, Kubo K, Yamamoto Y, et al. Alteration in gut environment accelerates emphysematous lesions by cigarette smoke in rats discontinuously fed with a fiber-free diet. Am J Respir Crit Care Med. 2014;189:A3000.

48. Tomoda K, Kubo K, Dairiki K, et al. Whey peptide-based enteral diet attenuated elastase-induced emphysema with increase in short chain fatty acids in mice. BMC Pulm Med. 2015;10(15):64.

49. Faner $\mathrm{R}, \mathrm{Cruz} T$, Agusti A. Immune response in chronic obstructive pulmonary disease. Expert Rev Clin Immunol. 2013;9(9):821-33.

50. Brusselle GG, Joos GF, Bracke KR, et al. New insights into the immunology of chronic obstructive pulmonary disease. Lancet. 2011;378(9795):1015-26.

51. Allinson JP, Hardy R, Donaldson GC, et al. The presence of chronic mucus hypersecretion across adult life in relation to chronic obstructive pulmonary disease development. Am J Respir Crit Care Med. 2016;193(6):662-72

\section{Publisher's Note}

Springer Nature remains neutral with regard to jurisdictional claims in published maps and institutional affiliations.
Ready to submit your research? Choose BMC and benefit from:

- fast, convenient online submission

- thorough peer review by experienced researchers in your field

- rapid publication on acceptance

- support for research data, including large and complex data types

- gold Open Access which fosters wider collaboration and increased citations

- maximum visibility for your research: over $100 \mathrm{M}$ website views per year

At BMC, research is always in progress.

Learn more biomedcentral.com/submissions 